\title{
Adnexal Masses in Pediatric and Adolescent Females: A Review of the Literature
}

\author{
R. N. Eskander • Robert E. Bristow
}

Published online: 31 December 2011

(C) Springer Science+Business Media, LLC 2011

\begin{abstract}
Adnexal masses are uncommon in the pediatric and adolescent population. The differential diagnosis includes ovarian lesions, tubal or paratubal lesions, nongynecologic lesions, and lesions related to infection or pregnancy. Presenting symptoms vary and may include acute abdominal pain, mass effect, and less commonly, precocious puberty and vaginal bleeding. Most of these lesions represent benign pathology, but malignant lesions are identified in a small subset of patients, and appropriate surgical and postoperative management is essential. It is important for clinicians caring for these patients to understand the differential diagnosis of an adnexal mass, to facilitate correct management (whether surgical or nonsurgical) and necessary referrals where appropriate. This review explores the etiologies, presenting symptoms, and appropriate management options for pediatric and adolescent patients with adnexal masses. There are few clinical trials guiding the management of these patients, and existing data comprise primarily case series and retrospective studies.
\end{abstract}

Keywords Adnexal mass · Adolescent · Benign · Malignant · Pediatric $\cdot$ Surgery $\cdot$ Torsion $\cdot$ Symptoms . Treatment $\cdot$ Management $\cdot$ Differential diagnosis $\cdot$ Pelvic

\section{R. N. Eskander $(\bowtie)$}

Department of Obstetrics and Gynecology, Division of

Gynecologic Oncology, University of California, Irvine,

101 The City Drive, Bldg 56 Suite 260,

Orange, CA 92868, USA

e-mail: eskander@uci.edu

\section{R. E. Bristow}

Department of OB/GYN, Division of Gynecologic Oncology, University of California Irvine Medical Center,

101 The City Drive, Bldg 56, Rm 260,

Orange, CA 92868, USA

e-mail: rbristow@uci.edu inflammatory disease $\cdot$ Ectopic pregnancy $\cdot$ Müllerian anomalies · Ovarian cyst · Paratubal cyst · Hydrosalpinx . Endometrioma $\cdot$ Cystic teratoma $\cdot$ Cystadenoma $\cdot$ Ovarian torsion . Ovarian cancer

\section{Introduction}

Adnexal masses requiring surgical intervention are uncommon in the pediatric population, with previous authors quoting 2.6 cases per 100,000 girls [1]. It is difficult to quantify the actual incidence of these lesions, but it is thought that $9 \%$ to $11 \%$ of adnexal masses in this age group are malignant [2]. Thus, most adolescent adnexal lesions are associated with benign pathology, including functional cysts and various benign neoplasms.

The presenting symptoms associated with an adnexal mass in pediatric patients vary; they include acute abdominal pain, mass effect, nausea, vomiting, and (less commonly) precocious puberty and vaginal bleeding [3]. The differential diagnosis includes benign and malignant ovarian lesions, tubal and paratubal cysts, müllerian abnormalities, disorders with infectious etiologies (pelvic inflammatory disease, PID), and pregnancy-related masses (ectopic pregnancy) (Table 1). In certain instances, such as for functional ovarian cysts, conservative management with observation alone is indicated, whereas operative management in the form of a simple cystectomy is sometimes needed. Minimally invasive modified cyst aspiration techniques also have been described [4].

When malignancy is identified, the Children's Oncology Group (COG) currently has several recommendations: (1) intact removal of the tumor without violation in situ; (2) sparing of the fallopian tube if not adherent; (3) obtaining ascites for cytology; (4) examination and palpation of the 
Table 1 Differential diagnosis of adnexal masses in pediatric and adolescent patients

\begin{tabular}{lll}
\hline & Benign & Malignant \\
\hline Gynecologic & Functional cyst & Germ cell tumors \\
& Corpus luteum cyst & Sex cord-stromal tumors \\
& Hydrosalpinx & Epithelial ovarian tumors \\
& Tubal/paratubal cysts & Metastatic tumors \\
& Endometrioma & \\
Mature cystic teratoma & \\
Cystadenoma & \\
Ectopic pregnancy & \\
& Pelvic inflammatory disease & \\
& Tubo-ovarian abscess & \\
& Müllerian anomalies & \\
Nongynecologic & Peritoneal inclusion cysts & \\
& Appendicitis/appendiceal abscess
\end{tabular}

omentum, with biopsy or removal of suspicious areas; and (5) examination and palpation of the iliac and aortocaval nodes, with biopsy of abnormal areas [5].

Given the rarity of malignant lesions, avoiding overly aggressive surgery is important. Adolescent patients receiving a unilateral salpingo-oophorectomy have a $3 \%$ to $15 \%$ lifetime risk of torsion or neoplasia in the contralateral ovary and are more commonly referred for infertility evaluation [6,7]. This subset of patients would subsequently be at risk of premature castration.

\section{General Considerations}

\section{Diagnosis}

The differential diagnosis of adnexal masses in pediatric and adolescent females is broad. Thus, a detailed and accurate history and physical examination is important. Specifically, the clinician should inquire about presenting symptoms and the duration and nature of the pain. Acute onset of sharp pain may represent adnexal torsion, whereas cyclic discomfort in a menstruating female may indicate endometriosis or müllerian anomalies. Furthermore, adolescents should be asked about sexual activity, including details of contraceptive practices, history of sexually transmitted diseases, and possible abuse.

Examination of a virginal pediatric patient may be limited, but abdominal palpation and rectal examination can assist in diagnosis. If a more detailed physical examination is warranted, examination under anesthesia should be considered. In addition, imaging in the form of abdominal/pelvic ultrasound, CT, or MRI should be considered.

Several ultrasound-based algorithms have been developed to assist in the differentiation between benign and malignant ovarian lesions in adult patients, with sensitivity ranging from $82 \%$ to $91 \%$ and specificity ranging from $68 \%$ to $83 \%$ [8 ]. Most ovarian tumors in patients less than 20 years of age are not epithelial in origin, however, and these algorithms have not been studied in this population [9•]. It has been shown that malignant lesions are significantly larger than their benign counterparts in pediatric and adolescent patients. In a recent study, malignant ovarian tumors measured $17.3 \pm 7.1 \mathrm{~cm}$ versus $8.8 \pm 7.1 \mathrm{~cm}$ for benign masses $(P<0.001)$ [10 $]$. Nonetheless, the experience obtained from studying adult patients with adnexal masses has been important in shaping the use of ultrasound in pediatrics.

The primary utility of ultrasound is its ability to distinguish simple cystic structures, which are nearly all benign, from solid or complex masses, for which surgical intervention may be warranted [6]. In addition, ultrasound imaging can assist in discriminating adnexal masses from gastrointestinal and uterine pathology.

If additional imaging is required or there is concern about likely malignancy, CT scanning and/or MRI can be used. The benefits of MRI are the lack of ionizing radiation exposure and its ability to better differentiate soft tissue boundaries, which may be important in patients with suspected müllerian anomalies. These imaging modalities may also be employed in patients who cannot tolerate a transvaginal ultrasound or examination.

In addition to imaging, laboratory studies may also assist in diagnosis, although they are nonspecific. Tumor markers, including beta-human chorionic gonadotropin ( $\beta$-hCG), alpha fetoprotein (AFP), lactate dehydrogenase (LDH), Inhibin, carcinoembryonic antigen (CEA), cancer antigen 125 (CA 125), and cancer antigen 19-9 (CA 19-9) can be obtained (Table 2). Significantly elevated CA 125 levels have been described in patients with a noncommunicating uterine horn and in those with torsed adnexa [11].

\section{Operative Management}

Operative management of adnexal masses in pediatric and adolescent females can be performed via laparotomy or

Table 2 Tumor markers and germ cell tumors of the ovary

\begin{tabular}{lcccc}
\hline & AFP & $\beta-\mathrm{hCG}$ & CA 19-9 & LDH \\
\hline Dysgerminoma & - & - & - & + \\
Choriocarcinoma & - & + & - & - \\
Immature teratoma & - & - & + & - \\
Endodermal sinus tumor & + & - & - & - \\
Embryonal carcinoma & + & + & - & - \\
\hline
\end{tabular}

$A F P$ alpha fetoprotein; $\beta-h C G$ beta-human chorionic gonadotropin; $C A$ 19-9 cancer antigen 19-9; $L D H$ lactate dehydrogenase 
minimally invasive laparoscopic techniques. If an ovarian malignancy is suspected preoperatively, laparotomy is recommended, to facilitate complete surgical staging. The importance of comprehensive staging in patients with malignant ovarian germ cell tumors was described by Palenzuela et al. [12]. The rate of improper surgical staging was higher for surgeons less experienced in gynecologic surgery. Furthermore, the risk of relapse in patients undergoing observation increased significantly if the tumor was improperly staged. The impact of appropriate staging on adjuvant therapy has also been studied, as observation without adjuvant chemotherapy may be appropriate for patients with early-stage disease. Taking these points into account, both laparotomy and consideration of a preoperative or intra-operative consultation with an experienced gynecologic cancer specialist are clearly indicated for patients in whom malignancy is encountered. In a recent study, the relative risk of incomplete surgical staging of malignant lesions was significantly reduced in the presence of a gynecologic oncologist (RR, 0.14; 95\% CI, 0.02-0.89; $P=0.003)[10 \bullet$.

In addition to the above indications, laparotomy may be required for patients with benign lesions who exhibit hemodynamic instability, who are known to have severe pelvic adhesive disease, or who have medical comorbidities such as cardiac or pulmonary disease, which may preclude abdominal insufflation. Mini-laparotomy may be used in such cases, allowing for improved cosmetic results.

Given the rarity of malignant lesions in pediatric and adolescent females, the laparoscopic approach has become routine. The benefits of laparoscopy include shortened recovery times, avoidance of overnight hospital stays, decreased pain, and improved cosmesis. Ultimately, preoperative evaluation and clinical judgment should guide the operative approach. New, modified cyst aspiration techniques have been described that allow laparoscopic management of cystic lesions as large as $25 \mathrm{~cm}$. It is important to remember, however, that if malignancy is diagnosed, spillage of cyst contents into the peritoneal cavity upstages patients from IA to IC, resulting in the need for adjuvant treatment.

Regardless of the approach employed, fertility preservation should be prioritized when possible. Historically, pediatric patients with adnexal masses were treated using oophorectomy and unilateral salpingo-oophorectomy, which can have an adverse impact on the fertility potential of these patients, as already noted. In 1999, Quint and Smith reported a salpingo-oophorectomy rate of $85 \%$, with nine patients undergoing bilateral oophorectomy [2]. More recently, ovarian preservation has been advocated. In 2001, Cass et al. showed a $39.6 \%$ ovarian conservation rate in pediatric patients undergoing surgery for the treatment of a benign ovarian mass [3]. In a recent retrospective chart review, the overall ovarian preservation rate approached
$50 \%$, with malignancy leading to the complete removal of the affected ovary in all 17 cases [10•].

Furthermore, an association has been identified between surgeon specialty and surgical outcome [13]. Templeman et al. investigated the management of noninflammatory ovarian masses in girls and found that gynecologists performed $70 \%$ of surgeries after 1989, compared with $90 \%$ in earlier periods; the ovarian preservation rate ranged from $60 \%$ to $64 \%[14$, 15]. Bristow et al. [13] also explored the impact of surgeon specialty on ovary-conserving surgery in young females with adnexal masses. In a retrospective review of 82 cases, the presence of a gynecologic surgeon was significantly and independently associated with ovarian preservation (OR, 8.71; 95\% CI, 2.12-41.41; $P=0.001)$.

\section{Selected Disorders}

\section{Inflammatory Disease}

\section{Pelvic Inflammatory Disease and Tubo-Ovarian Abscess}

More than 1 million women in the United States are affected with PID each year, according to the Centers for Disease Control and Prevention (CDC) [16]. The adolescent population is particularly susceptible to PID because of cervical ectopy and behavioral risk factors. In previous studies, up to $19 \%$ of adolescents diagnosed with PID were found to have a tubo-ovarian abscess (TOA) on ultrasound [17]. Symptoms of PID include abdominal/pelvic pain, cervicitis, cervical motion tenderness, leukocytosis, and fever. On imaging, tubo-ovarian abscesses are complex, thick-walled, and multi-septated, with echogenic internal debris $[8 \bullet]$. Current management recommendations per the $\mathrm{CDC}$ treatment guidelines include empiric broad spectrum antibiotic coverage of likely pathogens: cefotetan $2 \mathrm{~g}$ IV every $12 \mathrm{~h}$ or alternatively cefoxitin 2 g IV every $6 \mathrm{~h}$ plus doxycycline $100 \mathrm{mg}$ orally or IV every $12 \mathrm{~h}$ (2010 CDC treatment guidelines). Patients who fail to have a clinical response to IV antibiotics or who experience mass rupture may require surgical exploration or drainage, using interventional radiology, to facilitate resolution. Unfortunately, despite treatment, these patients can experience significant reproductive health sequelae, with up to $43 \%$ of these patients having chronic abdominal pain, 19\% experiencing infertility, and $21 \%$ having recurrent episodes of PID [16].

Pregnancy-Related Masses

\section{Ectopic Pregnancy}

Any menstruating, sexually active, adolescent female presenting with complaints of abdominal pain and/or 
irregular vaginal bleeding should have a urine or serum pregnancy test. If the pregnancy test is positive, ectopic pregnancy must be included in the differential diagnosis. Subsequently, these patients should have a quantitative serum $\beta$-hCG level drawn and a pelvic ultrasound performed. If the $\beta-\mathrm{hCG}$ is above the "discriminatory zone" $(1,500-2,000 \mathrm{mIU} / \mathrm{mL}$ for transvaginal ultrasound and $6,000 \mathrm{mIU} / \mathrm{mL}$ for transabdominal ultrasound) and no evidence of an intrauterine pregnancy exists, suspicion of an ectopic pregnancy should be high. Unfortunately, the ultrasound fails to establish a conclusive diagnosis in $8 \%$ to $31 \%$ of women with suspected complications of early pregnancy [18]. The differential diagnosis includes ectopic pregnancy, early intrauterine pregnancy, or nonviable intrauterine pregnancy/miscarriage. If suspicion is high, a uterine curettage is performed, and if no products of conception are identified, laparoscopy is usually warranted. Management may include a salpingostomy with evacuation of the tubal pregnancy or complete salpingectomy. Alternatively, in medically stable patients satisfying selection criteria, medical management with methotrexate delivered intramuscularly may be used. It is important to ensure appropriate follow-up of adolescent patients treated with methotrexate, as serial $\beta$-hCG measurements are required to confirm resolution. Appropriate contraception during this time should be emphasized. Lastly, in a small subset of patients with very low $\beta$-hCG values, observation alone has been shown to be appropriate.

\section{Müllerian Anomalies}

Müllerian anomalies affect up to $7 \%$ of the female population [19]. These patients have varying clinical presentations, with amenorrhea and cyclic pelvic pain being the most common. In patients with complete or partial obstruction of menstrual blood, adnexal masses may develop. The more common abnormalities resulting in these symptoms include vaginal agenesis, segmental vaginal atresia, transverse vaginal septum, uterine didelphys with obstructed hemivagina, unicornuate uterus with a noncommunicating rudimentary horn, or imperforate hymen. Although MRI has been shown to be $100 \%$ sensitive and $83 \%$ to $100 \%$ specific in diagnosing müllerian anomalies, laparoscopy may be required to adequately assess uterine contour and guide future management [20].

Patients with müllerian anomalies often require extensive counseling with a multidisciplinary approach (e.g., including a reproductive endocrinologist). Surgical interventions may be complicated, and patients must be emotionally mature (and in certain instances, sexually active) prior to intervention. Because of the complexity and rarity of many of these conditions, referral to an experienced center and surgeon is warranted.
Benign Lesions

\section{Functional Ovarian Cysts}

The most common benign adnexal lesions identified in pediatric and adolescent patients are functional physiologic ovarian cysts or corpus luteum cysts. In a large retrospective review, van Winter et al. [21] evaluated the clinical presentation and histopathologic findings of benign and malignant adnexal masses in infants, children, and adolescents. Between 1955 and 1992, 486 patients (aged 7 days through 20 years) with adnexal masses were surgically evaluated, and of 521 adnexal masses, 261 were functional or simple cysts and 143 were corpus luteum cysts.

During the menstrual cycle, ovarian primordial follicles respond to follicle stimulating hormone (FSH) by gradually enlarging, and ultimately, one dominant follicle measuring 1.8 to $2.0 \mathrm{~cm}$ develops [22]. Ovulation, which occurs approximately 14 days after the first day of the last menstrual period, results in involution of the dominant follicle. At times, however, the dominant follicle may have an excessive response to FSH stimulation and fail to involute, resulting in a persistent, simple-appearing adnexal cyst measuring 4 to $5 \mathrm{~cm}$. As these lesions are benign and generally regress spontaneously over several weeks, observation and surveillance are recommended.

Following ovulation, a corpus luteum cyst, measuring 2 to $3 \mathrm{~cm}$, is normally formed from remnants of the follicle. At times, these cysts can measure 4 to $5 \mathrm{~cm}$ and may appear heterogeneous or complex on imaging, owing to blood contents. Once again, observation is warranted, as most of these will resolve without intervention over one or two menstrual cycles. Uncommonly, a hemorrhagic corpus luteum cyst may result in significant pain or active bleeding, requiring surgical intervention.

In a large study conducted by Spanos [23], unilocular, mobile cystic masses ranging from $4 \mathrm{~cm}$ to $10 \mathrm{~cm}$ in size resolved within 6 weeks in 205 of 286 patients. Thus, observation is warranted for such patients unless the lesion persists for longer than 3 months, is enlarging, is larger than $8 \mathrm{~cm}$, or is associated with significant symptoms. It is important to note that use of oral contraceptives in patients with simple cysts has not been shown to hasten resolution of the mass [24].

\section{Tubal, Paratubal, and Peritoneal Inclusion Cysts}

Tubal, paratubal, and peritoneal inclusion cysts are normally identified incidentally on routine examination, or at the time of imaging in adolescent patients. They rarely result in pelvic pain due to rupture or torsion, with a reported isolated tubal torsion rate of 1 per 1.5 million [25]. These paratubal cysts are commonly $(76 \%)$ tubal/paramesonephric 
in origin, but they may be mesonephric in origin. Unless the patient is symptomatic, intervention to treat these simple cystic lesions is not warranted.

Peritoneal inclusion cysts traditionally arise in postmenarchal adolescents. These patients typically have active ovaries and concomitant pelvic adhesions, resulting in impaired absorption of peritoneal fluid. It is hypothesized that the adhesions develop after an inflammatory insult or after surgery, trapping peritoneal or ovarian fluid within them. Presenting symptoms often include progressive abdominal or pelvic pain, a palpable abdominal mass, or both, with symptom duration varying between weeks and months [26]. On ultrasound imaging, the lesions appear as anechoic cystic structures within the pelvis, adjacent to or adherent to neighboring pelvic organs, including the uterus or ovary. Occasionally, the adhesions may mimic thin-walled septations. Treatment options include observation for the asymptomatic patient with an incidentally discovered cyst, treatment with oral contraceptives to decrease cyst fluid accumulation, or surgical management in the form of cyst aspiration, sclerotherapy, or complete excision [27].

\section{Hydrosalpinx}

Hydrosalpinx is defined as a blocked, dilated, fluid-filled fallopian tube, which results from occlusion of the distal fimbriated end due to inflammatory or infectious processes or endometriosis [28]. Hydrosalpinx is uncommon in adolescent females, and rarely results in isolated tubal torsion, as described above. On imaging, the dilated, fluidfilled structure appears sausage-shaped [8•]. At times, the dilated tube may contain blood, resulting in a hematosalpinx, and the intratubal folds may be confused with mural nodules. In such instances, MRI can assist in diagnosis. In asymptomatic patients, observation is warranted, but surgical exploration is required if torsion is suspected or if the patient is symptomatic.

\section{Endometrioma}

In adolescent females experiencing chronic or cyclic pelvic pain, the incidence of endometriosis has been reported to be between $45 \%$ and $70 \%$ [29]. Endometriomas form when uterine glands and stroma are found in ovarian tissues and respond to hormonal stimulation. These implants result in formation of "chocolate cysts," which contain old blood and hemosiderin deposits within a cystic cavity lined with endometrium. On ultrasound imaging, endometriomas typically appear as unilocular or multilocular adnexal masses with low-level echoes, making it difficult to distinguish them from hemorrhagic cysts. Furthermore, these structures may at times contain mural nodules, requiring further imaging in the form of MRI to exclude malignancy [30]. As these cysts do not resolve spontaneously, cystectomy is recommended, with complete removal of the cyst wall to prevent recurrence.

\section{Mature Cystic Teratoma (Dermoid Cyst)}

In addition to the above lesions, mature cystic teratomas (dermoids) must also be considered in the evaluation of pediatric or adolescent adnexal masses. These are the most common benign ovarian neoplasms and are composed of totipotent cells that can give rise to ectoderm, mesoderm, and endoderm. Dermoids have a characteristic appearance on ultrasound imaging: (1) fat-fluid levels; (2) appearance of a "white ball" within part of the lesion, representing a dermoid plug; (3) "tip of the iceberg" appearance, representing a diffuse or partly echogenic mass; and (4) a "dermoid mesh" consisting of long, echogenic lines that represent hair floating within the cyst [8•]. Dermoid cysts do not resolve spontaneously and thus warrant surgical removal. Importantly, up to $7 \%$ of these lesions can be bilateral, so examination of the contralateral ovary is warranted, with cystectomy performed if indicated.

\section{Cystadenomas}

Benign serous or mucinous cystadenomas are uncommon neoplasms amongst pediatric and adolescent females. The only data come from case reports and retrospective series, in which the reported incidence ranges from approximately $1.4 \%$ to $2.6 \%[10 \bullet, 13]$. These lesions are usually large and at times may produce symptoms due to mass effect on adjacent pelvic and abdominal structures. Management consists of surgical intervention with cystectomy, if possible.

\section{Ovarian Torsion}

Ovarian torsion occurs when the ovary twists on its vascular support (the infundibulopelvic ligament, containing the ovarian artery and vein). Initially, venous obstruction occurs but arterial flow persists, resulting in an edematous and enlarged ovary. Ultimately, ovarian ischemia and necrosis occur. If diagnosis and management are delayed, loss of ovarian function may result. In a recent study conducted by Guthrie et al. [31], ovarian torsion and oophorectomy rates across the United States were estimated using the Kid's Inpatient Database for years 2000, 2003, and 2006. Based on the abstracted data, the annual incidence of ovarian torsion in females 1 to 20 years of age was estimated at 4.9 per 100,000 , making this a much less common diagnosis than appendicitis. A $60 \%$ oophorectomy rate was estimated. There was a $0.4 \%$ rate of 
malignant ovarian neoplasms in patients with torsion, and no diagnosis of thromboembolism was recorded in any hospitalization related to ovarian torsion.

The universal presenting symptom of adnexal torsion is abdominal pain, which may be episodic [32]. Unfortunately, presenting symptoms of pain, nausea, and emesis are nonspecific and can delay diagnosis, with acute appendicitis being the most common differential diagnosis [33]. Commonly, transvaginal ultrasound is used to evaluate blood flow to the ovary. However, blood flow on Doppler ultrasound scan does not rule out ovarian torsion; up to $64 \%$ of patients have arterial or venous flow [34]. Rather, ovarian enlargement has been reported as the most consistent ultrasound finding in patients with ovarian torsion. Despite advances in medical imaging, preoperative diagnosis remains uncertain. Clinical judgment (with a high index of suspicion) must be relied upon, as torsion is a surgical emergency.

When ovarian torsion occurs in pediatric and adolescent patients, ovarian conservation should be prioritized. Recent trends favor laparoscopic cystectomy and detorsion (untwisting of the pedicle) [35]. Cohen et al. [36] investigated outcomes after detorsion of necrotic or ischemic-appearing adnexa, and found ultrasound evidence of ovarian function in $93 \%$ of patients 3 months after surgery. Similarly, Aziz et al. [37] showed evidence of ovarian function in 14 of 17 patients following detorsion when intraoperative assessment showed ischemia.

\section{Malignant Lesions}

In pediatric and adolescent patients undergoing surgery for ovarian masses, the incidence of malignancy ranges from $4 \%$ to $9 \%$, accounting for $1 \%$ of all pediatric cancers [38]. Although ovarian neoplasms in children are rare, the diagnosis must be considered in any female pediatric patient with a pelvic mass.

\section{Germ Cell Tumors}

Ovarian germ cell tumors are diagnosed mostly in adolescents between 16 and 20 years of age. The presenting symptoms include abdominal pain due to rapid enlargement, hemorrhage, and mass effect [39]. In those patients found to have ovarian neoplasms, the incidence of germ cell tumors varies from $67 \%$ to $85 \%$, and nearly one third are malignant [40]. Dysgerminomas are the most common malignant germ cell tumors, representing $30 \%$ to $40 \%$ of all ovarian cancers of germ cell origin; $5 \%$ are discovered in phenotypic females with dysgenic gonads [41]. These are followed by immature teratomas, endodermal sinus tumors, embryonal carcinomas, choriocarcinomas, and polyembryomas, in order of decreasing frequency.
Fertility preservation is the standard of care for adolescent and pediatric patients diagnosed with ovarian germ cell tumors, unlike other types of ovarian cancer. The median age of affected patients is 19 , and the majority have stage I disease. The recommended management of young patients with suspected malignant germ cell tumors of the ovary includes (1) intact removal of the tumor (oophorectomy); (2) sparing of the fallopian tube, if not adherent to the tumor; (3) procurement of cytologic washings or harvesting of ascites fluid; (4) examination and palpation of the omentum, with removal of suspicious areas; and (5) examination and palpation of the pelvic and aortocaval nodes, with biopsy of abnormal areas [42]. With the use of postoperative systemic chemotherapy, $90 \%$ to $95 \%$ of malignant germ cell tumors of the ovary are curable [43]. Gershenson [44] described 40 patients treated with surgery and multiagent chemotherapy for malignant germ cell tumors of the ovary. The median age at the start of therapy was 15 years. All 28 patients treated with vincristine, doxorubicin, and cyclophosphamide (VAC) chemotherapy resumed regular menstrual function, with only 3 patients having persistent menstrual dysfunction. Of 16 patients attempting pregnancy, 11 delivered 22 healthy infants.

Currently, adjuvant chemotherapy is recommended for all patients except those with a stage IA, grade I immature teratoma. The currently recommended regimen of bleomycin, etoposide, and cisplatin (BEP) is derived from experience with more common testicular tumors, for which the etoposidecontaining regimen was shown to have a more favorable therapeutic index (particularly with regard to neurologic and gastrointestinal toxicities) [45]. In a trial randomizing $261 \mathrm{men}$ with disseminated germ cell tumors to vinblastine, bleomycin, and cisplatin (VBP) versus BEP, $74 \%$ of those receiving the VBP regimen and $83 \%$ of those receiving the BEP regimen became disease-free with or without subsequent surgery. Among 157 patients with high tumor volume, $61 \%$ became disease-free on VBP, compared with $77 \%$ on BEP $(P<0.05)$. Survival also was higher among the patients who received etoposide $(P=0.048)$. In addition, the etoposide regimen caused substantially fewer paresthesias $(P=0.02)$, abdominal cramps $(P=0.0008)$, and myalgias $(P=0.00002)$.

\section{Sex Cord-Stromal Tumors}

Sex cord-stromal tumors account for only $5 \%$ to $8 \%$ of all ovarian malignancies. This group of ovarian neoplasms includes granulosa cell tumors, thecomas, fibromas, SertoliLeydig cell tumors, and combinations of the various cell types. Granulosa cell tumors are seen in women of all ages, with up to $5 \%$ occurring in prepubertal females [46]. As these lesions can be hormonally active (most commonly secreting estrogen), they can result in precocious puberty, endometrial hyperplasia in $25 \%$ to $50 \%$ of cases, and endometrial cancer 
in up to $5 \%$ [47]. Fertility-preserving surgery should be prioritized, as outlined above for germ cell tumors.

Given the rarity of these malignancies, information regarding adjuvant chemotherapy for adolescent and pediatric patients with advanced stage sex cord-stromal tumors is limited. No prospective trials have been conducted, and it is difficult to draw conclusions regarding optimal therapy from retrospective trials because of small patient numbers and varying treatment regimens. Nonetheless, several regimens have been explored. Response rates to combination Adriamycin-cisplatin (AP), cyclophosphamide-Adriamycincisplatin (CAP), cisplatin-vinblastine-bleomycin (PVB), and bleomycin-etoposide-cisplatin (BEP) range from $37 \%$ to $100 \%$ in patients with advanced-stage or recurrent disease [48]. In addition, platinum and taxane combinations have shown activity in sex cord-stromal tumors and may have equivalent efficacy with lower toxicities when compared with BEP. It is our practice to treat these patients with either $\mathrm{BEP}$ or a platinum/taxane combination regimen for three to six cycles.

\section{Tumors of Low Malignant Potential and Epithelial Ovarian Cancers}

Epithelial ovarian cancer is uncommon in pediatric and adolescent patients. Most of these lesions are comprised of low malignant potential (LMP) tumors. In a retrospective review, You et al. [49] described the distribution of gynecologic cancers in patients less than 25 years of age. The most common primary site was the ovary $(46 \%$ of patients). Of those patients with epithelial ovarian lesions, nearly $70 \%$ were LMP tumors. When an LMP tumor is identified in an adolescent female, surgical management may be limited to unilateral salpingo-oophorectomy with complete surgical staging, provided that the tumor appears confined to one ovary [50]. However, patients should be counseled regarding recurrence rates greater than $30 \%$. If there is bilateral ovarian involvement and complete resection can be achieved, ovarian cystectomy is the treatment of choice [51]. Complete surgical staging-including exploration of the entire abdominal cavity, peritoneal washings, infracolic omentectomy, and multiple peritoneal biopsiesis important, as $20 \%$ of patients may have noninvasive as well as invasive metastatic implants [52].

Treatment of invasive epithelial ovarian cancer in adolescent patients adheres to management principles established for adult patients, with additional emphasis on fertility preservation if possible. In patients with welldifferentiated, encapsulated, unilateral lesions without adhesions or ascites, fertility-preserving surgery in the form of a unilateral salpingo-oophorectomy and complete staging, with preservation of the uterus and contralateral ovary, may be considered $[50,53]$. It is recommended that biopsy of a normal-appearing contralateral ovary be avoided, as this can result in mechanical infertility. If the contralateral ovary appears grossly normal, the risk of occult malignancy is less than $3 \%$. Adjuvant chemotherapy follows surgery in all patients except those with completely staged lesions of FIGO 1A grade 1 or $1 \mathrm{~B}$ grade 1.

\section{Conclusions}

The etiology of adnexal masses in pediatric and adolescent patients is broad. If surgery is performed, techniques of ovarian preservation should be prioritized when benign masses are encountered, to optimize future fertility potential. Conversely, when ovarian malignancy is identified, complete excision of the mass and accurate surgical staging is essential, in order to improve patient outcomes and guide adjuvant therapy. A collaborative approach, incorporating gynecologic surgeons as well as the pediatric surgeons who commonly care for this unique patient population, is paramount to the accomplishment of these goals.

Disclosure No potential conflicts of interest relevant to this article were reported.

\section{References}

Recently published papers of interest have been highlighted as

- Of importance

1. Skinner M, Schlatter M, Heifetz S, Grosfeld J. Ovarian neoplasms in children. Arch Surg. 1993;128(8):849-53. discussion 53-4.

2. Quint E, Smith Y. Ovarian surgery in premenarchal girls. J Pediatr Adolesc Gynecol. 1999;12(1):27-9.

3. Cass D, Hawkins E, Brandt M, Chintagumpala M, Bloss R, Milewicz A, et al. Surgery for ovarian masses in infants, children, and adolescents: 102 consecutive patients treated in a 15 -year period. J Pediatr Surg. 2001;36(5):693-9.

4. Ehrlich P, Teitelbaum D, Hirschl R, Rescorla F. Excision of large cystic ovarian tumors: combining minimal invasive surgery techniques and cancer surgery-the best of both worlds. J Pediatr Surg. 2007;42(5):890-3.

5. Billmire D. Germ cell tumors. Surg Clin North Am. 2006;86 (2):489-503. xi.

6. Pfeifer S, Gosman G. Evaluation of adnexal masses in adolescents. Pediatr Clin North Am. 1999;46(3):573-92.

7. Lass A. The fertility potential of women with a single ovary. Hum Reprod Update. 1999;5(5):546-50.

8. - Griffin N, Grant LA, Sala E. Adnexal masses: characterization and imaging strategies. Semin Ultrasound CT MR 2010;31 (5):330-46. This review outlines the different imaging modalities available to characterize adnexal masses and includes a suggested imaging algorithm.

9. - Stankovic ZB, Bjelica A, Djukic MK, Savic D. Value of ultrasonographic detection of normal ovarian tissue in the 
differential diagnosis of adnexal masses in pediatric patients. Ultrasound Obstet Gynecol 2010;36(1):88-92. The presence of normal ovarian tissue adjacent to an ovarian mass may assist in excluding malignant tumors in pediatric and adolescent patients.

10. • Eskander RN, Bristow RE, Saenz NC, Saenz CC. A retrospective review of the effect of surgeon specialty on the management of 190 benign and malignant pediatric and adolescent adnexal masses. J Pediatr Adolesc Gynecol 2011;24(5):282-5. Complete surgical staging in cases of malignant pediatric ovarian cancers is significantly more common in the presence of a gynecologic oncologist.

11. McCarthy JD, Erickson KM, Smith YR, Quint EH. Premenarchal ovarian torsion and elevated CA-125. J Pediatr Adolesc Gynecol. 2010;23(1):e47-50.

12. Palenzuela G, Martin E, Meunier A, Beuzeboc P, Laurence V, Orbach D, et al. Comprehensive staging allows for excellent outcome in patients with localized malignant germ cell tumor of the ovary. Ann Surg. 2008;248(5):836-41.

13. Bristow R, Nugent A, Zahurak M, Khouzhami V, Fox H. Impact of surgeon specialty on ovarian-conserving surgery in young females with an adnexal mass. J Adolesc Health. 2006;39(3):411-6.

14. Templeman C, Fallat M, Blinchevsky A, Hertweck S. Noninflammatory ovarian masses in girls and young women. Obstet Gynecol. 2000;96(2):229-33.

15. Piippo S, Mustaniemi L, Lenko H, Aine R, Mäenpää J. Surgery for ovarian masses during childhood and adolescence: a report of 79 cases. J Pediatr Adolesc Gynecol. 1999;12(4):223-7.

16. Trent M, Haggerty CL, Jennings JM, Lee S, Bass DC, Ness R. Adverse adolescent reproductive health outcomes after pelvic inflammatory disease. Arch Pediatr Adolesc Med. 2011;165(1):49-54.

17. Golden N, Neuhoff S, Cohen H. Pelvic inflammatory disease in adolescents. J Pediatr. 1989;114(1):138-43.

18. Jurkovic D, Wilkinson H. Diagnosis and management of ectopic pregnancy. BMJ. 2011;342:d3397.

19. Grimbizis GF, Campo R. Congenital malformations of the female genital tract: the need for a new classification system. Fertil Steril. 2010;94(2):401-7.

20. Kimble RM, Khoo SK, Baartz D. The obstructed hemivagina, ipsilateral renal anomaly, uterus didelphys triad. Aust N Z J Obstet Gynaecol. 2009;49(5):554-7.

21. van Winter JT, Simmons PS, Podratz KC. Surgically treated adnexal masses in infancy, childhood, and adolescence. Am J Obstet Gynecol. 1994;170(6):1780-6. discussion 6-9.

22. Powell JK. Benign adnexal masses in the adolescent. Adolesc Med Clin. 2004;15(3):535-47.

23. Spanos WJ. Preoperative hormonal therapy of cystic adnexal masses. Am J Obstet Gynecol. 1973;116(4):551-6.

24. Steinkampf MP, Hammond KR, Blackwell RE. Hormonal treatment of functional ovarian cysts: a randomized, prospective study. Fertil Steril. 1990;54(5):775-7.

25. Seshadri S, Morris A, Uchil D, Joloaso A. Bilateral paratubal cysts with co-existent fallopian tube torsion in an adolescent. J Obstet Gynaecol. 2009;29(6):564-5.

26. Weiss SW, Tavassoli FA. Multicystic mesothelioma. An analysis of pathologic findings and biologic behavior in 37 cases. Am J Surg Pathol. 1988;12(10):737-46.

27. Vallerie AM, Lerner JP, Wright JD, Baxi LV. Peritoneal inclusion cysts: a review. Obstet Gynecol Surv. 2009;64(5):321-34.

28. Merlini L, Anooshiravani M, Vunda A, Borzani I, Napolitano M, Hanquinet S. Noninflammatory fallopian tube pathology in children. Pediatr Radiol. 2008;38(12):1330-7.

29. Laufer MR, Goitein L, Bush M, Cramer DW, Emans SJ. Prevalence of endometriosis in adolescent girls with chronic pelvic pain not responding to conventional therapy. J Pediatr Adolesc Gynecol. 1997;10(4):199-202.
30. Asch E, Levine D. Variations in appearance of endometriomas. J Ultrasound Med. 2007;26(8):993-1002.

31. Guthrie BD, Adler MD, Powell EC. Incidence and trends of pediatric ovarian torsion hospitalizations in the United States, 2000-2006. Pediatrics. 2010;125(3):532-8.

32. Chang YJ, Yan DC, Kong MS, Wu CT, Chao HC, Luo CC, et al. Adnexal torsion in children. Pediatr Emerg Care. 2008;24(8):534-7.

33. Emonts M, Doornewaard H, Admiraal JC. Adnexal torsion in very young girls: diagnostic pitfalls. Eur J Obstet Gynecol Reprod Biol. 2004;116(2):207-10.

34. Hurh PJ, Meyer JS, Shaaban A. Ultrasound of a torsed ovary: characteristic gray-scale appearance despite normal arterial and venous flow on Doppler. Pediatr Radiol. 2002;32(8):586-8.

35. Houry D, Abbott J. Ovarian torsion: a fifteen-year review. Ann Emerg Med. 2001;38(2):156-9.

36. Cohen S, Oelsner G, Seidman D, Admon D, Mashiach S, Goldenberg M. Laparoscopic detorsion allows sparing of the twisted ischemic adnexa. J Am Assoc Gynecol Laparosc. 1999;6(2):139-43.

37. Aziz D, Davis V, Allen L, Langer J. Ovarian torsion in children: is oophorectomy necessary? J Pediatr Surg. 2004;39(5):750-3.

38. von Allmen D. Malignant lesions of the ovary in childhood. Semin Pediatr Surg. 2005;14(2):100-5.

39. Abu-Rustum NR, Aghajanian C. Management of malignant germ cell tumors of the ovary. Semin Oncol. 1998;25(2):235-42.

40. Breen J, Maxson W. Ovarian tumors in children and adolescents. Clin Obstet Gynecol. 1977;20(3):607-23.

41. Kurman RJ, Scardino PT, McIntire KR, Waldmann TA, Javadpour N, Norris HJ. Malignant germ cell tumors of the ovary and testis. An immunohistologic study of 69 cases. Ann Clin Lab Sci. 1979;9 (6):462-6.

42. Billmire D. Malignant germ cell tumors in childhood. Semin Pediatr Surg. 2006;15(1):30-6.

43. Perrin L, Low J, Nicklin J, Ward B, Crandon A. Fertility and ovarian function after conservative surgery for germ cell tumours of the ovary. Aust N Z J Obstet Gynaecol. 1999;39(2):243-5.

44. Gershenson D. Menstrual and reproductive function after treatment with combination chemotherapy for malignant ovarian germ cell tumors. J Clin Oncol. 1988;6(2):270-5.

45. Williams SD, Birch R, Einhorn LH, Irwin L, Greco FA, Loehrer PJ. Treatment of disseminated germ-cell tumors with cisplatin, bleomycin, and either vinblastine or etoposide. N Engl J Med. 1987;316 (23): $1435-40$

46. Segal R, DePetrillo AD, Thomas G. Clinical review of adult granulosa cell tumors of the ovary. Gynecol Oncol. 1995;56(3):338-44.

47. Aboud E. A review of granulosa cell tumours and thecomas of the ovary. Arch Gynecol Obstet. 1997;259(4):161-5.

48. Colombo N, Parma G, Zanagnolo V, Insinga A. Management of ovarian stromal cell tumors. J Clin Oncol. 2007;25(20):2944-51.

49. You W, Dainty LA, Rose GS, Krivak T, McHale MT, Olsen CH, et al. Gynecologic malignancies in women aged less than 25 years. Obstet Gynecol. 2005;105(6):1405-9.

50. National Comprehensive Cancer Network (NCCN). NCCN Guidelines Version 2.2011 Epithelial Ovarian Cancer/Fallopian Tube Cancer/Primary Peritoneal Cancer. Available at www.ncen. org. Accessed Dec. 12, 2010.

51. Rao G, Skinner E, Gehrig P, Duska L, Miller D, Schorge J. Fertility-sparing surgery for ovarian low malignant potential tumors. Gynecol Oncol. 2005;98(2):263-6.

52. Cadron I, Leunen K, Van Gorp T, Amant F, Neven P, Vergote I. Management of borderline ovarian neoplasms. J Clin Oncol. 2007;25(20):2928-37.

53. Tewari K, Di Saia P. Ovulatory failure, fertility preservation and reproductive strategies in the setting of gynecologic and nongynecologic malignancies. Eur J Gynaecol Oncol. 2006;27 (5):449-61. 